\title{
La metodología Flipped Classroom en la enseñanza híbrida universitaria: la satisfacción de los estudiantes Flipped Classroom Methodology for Hybrid Learning in the Higher Education Context: Students Satisfaction
}

\author{
Susana Sousa Santos ${ }^{1}$, María José Peset Gonzalez ${ }^{2}$, Jesús Muñoz Sepúlveda ${ }^{1}$ \\ susana.sousa@universidadeuropea.es, mariajose.peset@universidadeuropea.es, jesus.munoz@universidadeuropea.es \\ ${ }^{1}$ Departamento Global, Relaciones y Negocios Internacionales \\ Universidad Europea \\ Madrid, España \\ ${ }^{2}$ Departamento ADE
Universidad Europea
Madrid, España
}

\begin{abstract}
Resumen- Tras más de dos décadas de desarrollo de la enseñanza online, hace su aparición la enseñanza híbrida que busca, entre otros objetivos, mejorar los puntos débiles de la docencia exclusivamente online. La enseñanza híbrida necesita dotarse de metodologías e instrumentos que desarrollen todo su potencial didáctico y es en este contexto en el que se inscribe el presente trabajo. Hemos investigado la satisfacción de los estudiantes con la enseñanza híbrida en relación a la exclusivamente online, así como la percepción de los mismos en cuanto a la aplicación de la metodología Flipped Classroom (FC) en el ámbito híbrido en oposición a la metodología tradicional de clases presenciales magistrales. La investigación se ha desarrollado para ciencias sociales, en aulas híbridas universitarias, aplicando métodos cualitativos y cuantitativos, como encuestas y reuniones semiestructuradas con los alumnos, durante el segundo semestre del curso 2016/17. Las conclusiones obtenidas son muy alentadoras, mostrando un alto grado de satisfacción por parte de los estudiantes con el entorno híbrido en comparación al exclusivamente online y con la aplicación de la metodología FC en dicho ámbito, frente a metodologías más tradicionales.
\end{abstract}

Palabras clave: Flipped Classroom, Enseñanza Híbrida, Enseñanaza Universitaria, Satisfacción Estudiantes, Innovación Educativa

Abstract- After more than two decades of the development of online education, the recent emergence of hybrid education has tried to overcome the weaknesses of the teaching exclusively online. The main goal of this paper is to identify the best methodologies and instruments of the hybrid education in order to develop all its educational potential. In this regard, we have addressed the satisfaction of the students with the hybrid teaching format compared to the exclusively online, as well as their perception with the new Flipped Classroom (FC) methodology as opposed to the traditional methodology in which theory is explained in class and practice is implemented at home. The research has been carried out in university hybrid courses in social sciences and has used qualitative and quantitative methods, such as surveys and structured interviews or appointments with students during the second semester of the 2016/17 academic year. So far, the conclusions of the research are very encouraging. In particular, they show a high degree of students' satisfaction with the hybrid format compared to the exclusively $100 \%$ online and with the application of the FC methodology with respect to the traditional teaching methodology.
Keywords: Flipped Classroom, Hybrid Teaching,

Higher Education, Student Satisfaction, Educational Innovation.

\section{INTRODUCCIÓN}

El presente trabajo parte de la experiencia adquirida por un grupo de investigación-acción de profesores de la Universidad Europea de Madrid que ha venido trabajando en los últimos años en la aplicación de la metodología Flipped Classroom (FC) en su docencia. Por otra parte, la implementación de la enseñanza híbrida en esta Universidad ha provocado la necesidad de buscar metodologías adecuadas a este ambiente docente que permitan alcanzar los objetivos de aprendizaje. Por tanto, nuestra investigación se encuadra en dos ámbitos, la enseñanza híbrida en el ambiente universitario y la metodología FC o aula invertida aplicada a ésta, y su objetivo es doble: conocer la satisfacción de los alumnos de la enseñanza híbrida en relación a la impartida exclusivamente online y, por otra parte, evaluar la preferencia de estos mismos alumnos entre la metodología FC y la tradicional, en la que la clase presencial se utiliza para impartir conocimiento teórico y la práctica del mismo se realiza fuera del aula.

Los ambientes híbridos de aprendizaje son aquellos que combinan la instrucción face-to-face con la enseñanza a través de las tecnologías de la información y la comunicación (Osorio, 2010). Como muy acertadamente plantea Llorente y Cabero (2008) se trata de ámbitos de aprendizaje en los que lo presencial y lo virtual convergen a través de la combinación de espacios (tanto físicos como virtuales), tiempos (asincronía y sincronía) y recursos (analógicos y digitales), modificando los procesos de aprendizaje y enseñanza, así como los modelos organizativos. Para delimitar si un aprendizaje presencial se puede considerar híbrido, diversos autores (Allen et al., 2007; Garrison y Kanuka, 2004; Graham, 2006) utilizan como criterio discriminante el porcentaje de recursos online utilizados para transmitir conocimiento y/o actividades de evaluación del mismo. En nuestra opinión, es más acertado el planteamiento de Dziuban et al (2004) según el cual la enseñanza híbrida no se define por la cantidad de horas de trabajo online, sino que se trata de un planteamiento pedagógico que combina la efectividad y socialización que

Octubre 4-6, 2017, Zaragoza, ESPAÑA

IV Congreso Internacional sobre Aprendizaje, Innovación y Competitividad (CINAIC 2017) 
proporciona la clase presencial junto a las posibilidades de mejora del aprendizaje que procura el entorno online.

En ese sentido, la enseñanza híbrida trata de optimizar dos formas de aprendizaje que tienen su origen y desarrollo de forma independiente: la enseñanza presencial, que tradicionalmente ha venido utilizado las TIC únicamente como repositorio documental, y la enseñanza online, que no suele utilizar los beneficios del face-to-face en el aprendizaje. Ahora bien, es importante destacar que no se trata solo de introducir conjuntamente dos formas de enseñanza independientes en los programas educativos, sino que, como indica Duart et al (2008), el objetivo es conseguir un resultado de aprendizaje que es totalmente inseparable, partiendo de entornos de docencia claramente diferenciados. Esto es importante, porque supone un replanteamiento del diseño convencional de los programas formativos tanto presenciales como online. Dziuban et al (2004) avanza tres ejes de este rediseño: un nuevo planteamiento de las sesiones presenciales en las que los estudiantes se conviertan en elementos activos y proactivos de su aprendizaje, el incremento de la interacción en todos los ámbitos (estudiante-profesor, estudiante-estudiante, estudiante-contenidos, estudiante-fuentes externas) y mecanismos de evaluación que aúnen ambos entornos.

Arbaugh (2010) tras su revisión de la literatura sobre comparación entre enseñanza híbrida y online concluye que los entornos híbridos pueden producir resultados más positivos que los exclusivamente impartidos online debido a un mayor control sobre el aprendizaje y a la interacción con otros estudiantes.

Dentro de este replanteamiento del modelo pedagógico necesario para que la enseñanza híbrida alcance todo su potencial, entendemos que la metodología FC puede ser la más adecuada.

Los orígenes de esta metodología FC (Aula Invertida, o mejor, Aula Participativa) podemos situarlos en los años 90 del siglo pasado, principalmente a partir de los trabajos del profesor Mazur (1997) en la Universidad de Harvard.

Una primera definición de esta metodología sería la siguiente: "The flipped classroom is a new pedagogical method, which employs asynchronous video lectures and practice problems as homework, and active, group-based problem solving activities in the classroom" (Bishop y Verleger, 2016). La idea central implica, pues, una redistribución del tiempo que se dedicaba hasta ahora en los sistemas educativos convencionales y una mayor participación activa por parte del alumnado en el proceso de aprendizaje. El profesor difunde sus lecciones aprovechando el soporte online -apuntes escritos, presentaciones, vídeos, etc.-, los alumnos trabajan esa documentación fuera del aula y las clases presenciales se destinan al debate, aplicación práctica, resolución de dudas y trabajo colaborativo necesarios para el logro de los objetivos de aprendizaje. Se trata de una metodología flexible que permite su aplicación en distintos ámbitos: enseñanza secundaria o universitaria y con modelos presenciales, semipresenciales e incluso, aunque resulte algo forzado, en línea. Las posibles ventajas de FC son muy amplias: mayor cantidad de tiempo de los profesores para atender de forma individual a los alumnos, se mejora la relación alumno/profesor, posibilita el proceso de comunicación de información entre profesores y de éstos con los alumnos, permite a los alumnos volver a ver las lecciones cuantas veces necesiten, crea un ambiente colaborativo, no se producen retrasos en los alumnos que no pueden asistir a las clases presenciales e incrementa la responsabilidad del alumno en su aprendizaje, fomentando la experimentación y aplicación práctica de sus conocimientos (Aste, 2012).

No obstante, los resultados de la aplicación de esta metodología resultan dispares, como demuestra el estudio de Chen y Chen (2016), y dependen de distintos factores, como la personalidad y predisposición de cada alumno para adaptarse y colaborar con este sistema o la materia objeto de estudio. Çakiroglu y Öztürk (2017) observan que no todo tipo de estudios se adaptan a esta metodología. Por el momento, las experiencias realizadas se han producido en campos muy concretos de las ciencias exactas y aplicadas (Çakiroglu y Öztürk, 2017), de las ciencias sociales (Hernández Nanclares y Pérez Rodríguez, 2016; Wolff y Chan, 2016) y de las Humanidades (Chuang, Weng y Chen, 2016).

Como indican Rotellar, Pharm y Cain (2016), la razón de ser de esta metodología es aumentar el compromiso de los estudiantes con la materia, incrementar y mejorar el tiempo de contacto de los profesores con los estudiantes y desarrollar su aprendizaje. Si bien no todos los analistas están de acuerdo con sus aplicaciones y ello ha ocasionado ciertas polémicas, la mayor parte de los casos estudiados indican que este modelo ofrece ventajas respecto a los usos y costumbres tradicionales (Blair, Maharaj y Primus, 2016).

Esta metodología, que puede considerarse beneficiosa y progresista (Rutherfoord, 2013), requiere de una importante reflexión sobre los aspectos concretos de su implementación. Cuestión que encontramos en los ámbitos de estudio de nuestra investigación: España y EEUU.

En este sentido, la literatura sobre FC ha crecido mucho en los últimos tiempos, y los resultados de las investigaciones no siempre han sido coincidentes. La búsqueda de un modelo uniforme es el objetivo principal de la revisión de Zuber (2016) que recoge las publicaciones aparecidas entre los años 2013 y 2014, y que precisa, en consecuencia, ser actualizada.

En los actuales sistemas de enseñanza tan importante es la evaluación de los profesores a los alumnos como la de los alumnos a los profesores (Butt, 2014) y esta última debe servir para mejorar la implementación de los cursos. En este sentido, nuestro trabajo toma en consideración casos previos, como el trabajo realizado en la Universidad de Oviedo por parte de los profesores Hernández Nanclares y Pérez Rodríguez (2016) sobre satisfacción de los alumnos con la aplicación de FC en cursos bilingües. Sus principales hallazgos confirman la preferencia de los estudiantes por FC frente a la enseñanza tradicional y proponen avanzar en la investigación de los efectos de esta metodología en la motivación y participación de los alumnos.

Aprovechando nuestra dedicación a la actividad docente y nuestro compromiso con la innovación educativa, nos hemos propuesto dilucidar y responder a las objeciones planteadas acerca de la idoneidad de la metodología FC y demostrar la mayor operatividad de este sistema en el ámbito híbrido universitario frente al modelo exclusivamente online. Para ello hemos centrado nuestra investigación en el nivel de satisfacción de nuestros alumnos, sus causas y determinantes. 


\section{CONTEXTO}

Hemos considerado que la aplicación de la metodología FC en el ambiente híbrido permite un mayor y más interesante aprovechamiento de las horas en las que alumnos y profesor se encuentran, y en las que los alumnos tienen relación entre sí. Adicionalmente, posibilita extraer todo el potencial de ambiente online, como es su carácter asincrónico, que permite trabajar y visionar el material proporcionado cuando y cuantas veces se desee, así como una mayor diversidad de documentación y material que el exclusivamente proporcionado en las clases magistrales presenciales.

En este contexto hemos querido investigar cuál es el grado de satisfacción de los estudiantes en aulas hibridadas, definidas como aquellas que combinan horas de docencia presencial y online en un porcentaje similar, en las que se ha trabajado con metodología FC. Nuestro objetivo es, por una parte, conocer la satisfacción de nuestros estudiantes en el ámbito híbrido con respecto al $100 \%$ online y, por otra, averiguar cómo perciben la metodología utilizada.

En cuanto al primer objetivo, preguntamos a nuestros estudiantes si prefieren la enseñanza híbrida con respecto a aquella que se imparte exclusivamente en el entorno online, así como las posibles causas de su preferencia o no preferencia. En relación al segundo objetivo, consultamos a nuestros alumnos si prefieren la metodología FC frente a la tradicional, entendida esta última como aquella en la que la teoría se imparte en clase y la práctica se realiza fuera de ésta $\mathrm{y}$, también en este caso, les preguntamos sobre las posibles causas de esta preferencia o no preferencia.

Además de todo ello profundizamos en aspectos específicos del entorno híbrido, como son su opinión sobre la mejor proporción entre horas de enseñanza online y presencial en sus cursos híbridos y su percepción sobre su nivel de aprendizaje.

La investigación se ha planteado en una población universitaria que ha cursado estudios en aulas híbridas en las que se ha utilizado de forma mayoritaria (más de un $80 \%$ de las clases) la metodología FC. Se ha llevado a cabo un muestreo casual, sistema habitual en la investigación en el ámbito educativo, en estudios de Grado en España y en USA, durante los dos primeros trimestres del curso académico 2016/2017. La muestra está formada por 15 aulas de grado con una población de 220 estudiantes.

\section{DESCRIPCIÓN}

Para realizar esta investigación hemos partido del concepto desarrollado por Bisquerra et al (2012) para el cual "la investigación educativa está dirigida a la búsqueda sistemática de nuevos conocimientos con el fin de que estos sirvan de base tanto para la comprensión de los procesos educativos como para la mejora de la educación”. Nosotros nos hemos fijado como objetivo la segunda parte de esta definición y para ello desarrollamos la primera: queremos que nuestra investigación sirva para mejorar la educación y para ello vamos a tratar de entender mejor el proceso educativo.

Para ello hemos llevado a cabo una triangulación metodológica, utilizando tanto métodos cuantitativos como métodos cualitativos. Siguiendo a Aguilar y Barroso (2015) entendemos que estos métodos se complementan, de forma que su combinación permite beneficiarse de las fortalezas de ambos y reduce sus debilidades, cruzando datos para analizar la confluencia de conclusiones entre uno y otro.

En este proyecto se utilizan dos técnicas de recogida de información: la encuesta cerrada y la entrevista grupal semiestructurada. En cada uno de los trimestres (enero-marzo/2017 y abril-junio/2017) se ha llevado a cabo una encuesta a los alumnos con cuestionarios cerrados con una escala Likert con cuatro opciones (totalmente en desacuerdo, parcialmente en desacuerdo, parcialmente de acuerdo y totalmente de acuerdo), en los que partiendo de 14 preguntas principales se formulan hasta 58 cuestiones. La encuesta se realiza online en clase a través del programa e-NQUEST. La organización de las preguntas se refiere a los siguientes aspectos:

a) Preferencia entre la enseñanza híbrida con respecto a exclusivamente online, preferencia entre la metodología FC y la tradicional y, por último, la adecuación de la proporción de tiempo online y tiempo presencial en la enseñanza híbrida recibida. En estas tres preguntas se realizó un desdoblamiento para la siguiente cuestión en función de que el alumno hubiera estado de acuerdo (total o parcialmente) con la inicial o no hubiera estado de acuerdo (total o parcialmente), preguntando sobre las causas del mismo a través de diferentes posibles alternativas.

b) Características de su aprendizaje, comparando las aulas híbridas con las 100\% online y la metodología FC y la tradicional, en cuanto a su percepción sobre en cuál de los dos casos se aprende más y se obtiene mejores calificaciones.

Para completar la información obtenida de los cuestionarios se realizan grupos de discusión o focus group con estudiantes que hayan recibido formación en las aulas de la muestra. Siguiendo las consideraciones de Bisquerra (2012) sobre las características idóneas de este tipo de grupos, las reuniones son dirigidas por un moderador externo a los centros educativos que plantea preguntas abiertas y supervisa el desarrollo de la sesión. Las sesiones reúnen en torno a ocho alumnos y su duración no se extiende más allá de 90 minutos. Se realizan tres reuniones, dos de ellas con alumnos españoles y una con alumnos norteamericanos. Se elabora un guion para dirigir la discusión fundamentado en la estructura del cuestionario pero realizada en un ambiente más abierto, de forma que posibilite la introducción de comentarios y aspectos que completan la información facilitada por la encuesta.

\section{RESUltados}

Los resultados de la primera y la segunda ola de encuestas realizadas durante el segundo y el tercer trimestre del curso académico 2016/17 muestran una participación total de 164 estudiantes, de los cuales el 67,1\% se corresponde con encuestas realizadas por estudiantes de la Universidad Europea de Madrid y el 32,1\% restante con encuestas realizadas por estudiantes de Kendall College de Chicago. Para analizar el grado de satisfacción de la metodología FC, en la Universidad Europea de Madrid se eligieron once asignaturas pertenecientes a diferentes áreas de conocimiento: Introducción al Derecho Procesal, Derechos Reales II, Taller de práctica jurídica II, Contabilidad Financiera I, Contabilidad Financiera II, Organización Industrial, Comportamiento del Consumidor, Fiscalidad Individual, Fiscalidad Empresarial, Sistemas Impositivos y Gestión Financiera. En Kendall College de Chicago se seleccionaron cuatro asignaturas híbridas pertenecientes al área de General Education. Esta 
selección permite evaluar si la propia naturaleza de la asignatura afecta a los niveles de satisfacción obtenidos en la aplicación de esta metodología educativa. Tanto las encuestas como los focus group evidencian una mayor aceptación de la metodología en aquellas asignaturas que cuentan con un mayor contenido cuantitativo.

Para analizar el grado de satisfacción de los estudiantes con la nueva metodología FC y con el ambiente híbrido de aprendizaje, los estudiantes debían comenzar evaluando su nivel de acuerdo con las siguientes frases: "Prefiero trabajar en un entorno híbrido (presencial y online) que únicamente online" y "Me ha gustado el sistema de estudiar online los aspectos teóricos de la materia y resolver dudas y realizar prácticas en las sesiones presenciales”.

En relación con la primera afirmación, los resultados muestran que el $79,3 \%$ de los estudiantes encuestados prefieren trabajar en un entorno híbrido que únicamente online. Específicamente, el 51,2\% de los alumnos estuvieron totalmente de acuerdo con esta afirmación, mientras que el 28,1\% eligieron la opción parcialmente de acuerdo. Únicamente el 9,75\% mostraron un total desacuerdo con esta afirmación. Una vez definida la preferencia sobre la implantación de este entorno, los estudiantes debían indicar los principales motivos de su elección: mayor proximidad con el profesor, mejor resolución de las dudas, mayor capacidad de aprendizaje, mejor aprovechamiento del tiempo presencial y del tiempo del alumno, mayor autonomía y mayor participación activa en el aula. La Tabla 1 muestra los resultados del porcentaje de acuerdo o desacuerdo de cada una de las afirmaciones anteriores. Como se puede observar, los alumnos destacan que estudiar en el entorno híbrido les permite un mejor aprovechamiento del tiempo presencial y una mejor resolución de sus dudas, lo que, en general, les proporciona una mayor capacidad de aprendizaje.

Tabla 1

\begin{tabular}{l|c|c}
\hline \hline Causas de la preferencia & $\begin{array}{c}\text { Acuerdo } \\
(\mathbf{\%})\end{array}$ & $\begin{array}{c}\text { Desacuerdo } \\
\mathbf{( \% )}\end{array}$ \\
\hline Mejor aprovechamiento tiempo & 89,2 & 10,8 \\
presencial & 88,5 & 11,5 \\
Mejor resolución de las dudas & 86,2 & 13,8 \\
Mayor capacidad de aprendizaje & 86,2 & 13,8 \\
Mayor participación activa & 83,1 & 16,9 \\
Mayor proximidad al profesor & 73,1 & 26,9 \\
Mayor autonomía & 70,8 & 29,2 \\
Mejor aprovechamiento tiempo & del alumno &
\end{tabular}

Por otro lado, en el caso de la no preferencia de la enseñanza híbrida frente a la 100\% online también debían destacar las causas de su opinión: no haber entendido en qué consistía el entorno híbrido, la reducción de su autonomía, la suficiencia del entorno online para la resolución de dudas o la consideración de haber aprendido menos bajo este entorno. La Tabla 2 recoge los porcentajes sobre el nivel de acuerdo y desacuerdo de las afirmaciones anteriores en la muestra de alumnos considerada. Como puede observarse, los alumnos destacan el menor nivel de autonomía, así como la no comprensión de este entorno de aprendizaje, como las principales causas para no preferir el ambiente híbrido. En este sentido, sin embargo, es necesario destacar el bajo porcentaje de acuerdo de cada una de las causas que justifican su no preferencia por este entorno.

Tabla 2

\begin{tabular}{l|c|c}
\hline \hline Causas de la no preferencia & $\begin{array}{c}\text { Acuerdo } \\
\text { (\%) }\end{array}$ & $\begin{array}{c}\text { Desacuerdo } \\
\text { (\%) }\end{array}$ \\
\hline Menor autonomía & 38,2 & 61,8 \\
No comprensión del entorno & 35,3 & 64,7 \\
Menor nivel de aprendizaje & 32,4 & 67,6 \\
$\begin{array}{l}\text { La parte online es suficiente (sin } \\
\text { clases presenciales) }\end{array}$ & 29,4 & 70,6 \\
\hline \hline
\end{tabular}

Los resultados obtenidos sobre la potencial preferencia de este entorno de aprendizaje fueron ampliamente discutidos también en el focus group de alumnos, en el que volvieron a destacar las diferencias entre las materias más cuantitativas y las más teóricas, y que, en términos generales, consideran que se aprende más y mejor en un entorno híbrido que $100 \%$ online.

La segunda afirmación donde los estudiantes debían mostrar su nivel de acuerdo o desacuerdo pretendía evaluar el grado de satisfacción de los estudiantes con la aplicación de la metodología FC. Los resultados obtenidos revelan que el $66,4 \%$ de los alumnos parecen estar de acuerdo con la implantación de esta nueva metodología educativa. En concreto, el $21,3 \%$ y el $45,1 \%$ de los estudiantes encuestados estuvieron totalmente y parcialmente de acuerdo con dicha afirmación, respectivamente. A este respecto, solo el $13.4 \%$ muestra un total desacuerdo con la aplicación de esta metodología. Una vez identificada la preferencia de los estudiantes por esta metodología, el siguiente bloque de preguntas de la encuesta pretendía identificar las principales ventajas derivadas de la implantación de FC. Específicamente, los estudiantes debían mostrar su nivel de acuerdo con toda una serie de ventajas potenciales: mejor administración del tiempo de trabajo, mayor autonomía del estudiante, mayor confianza en el trabajo de los compañeros y mayor capacidad de aprendizaje. En general, la principal ventaja que identifican los alumnos está relacionada con la existencia de una mayor autonomía en el proceso de estudio y aprendizaje de la asignatura. En cuanto a los que no están satisfechos con la metodología FC, las principales causas expresadas son la preferencia por metodologías más tradicionales y la mayor carga de trabajo. En el focus group, los alumnos consideraron relevantes en la impartición de esta metodología aspectos como la adecuada organización de las clases presenciales, la importancia de utilizar éstas para aclarar dudas y la calidad de los materiales para estudiar fuera del aula presencial.

Por último, y vinculado con el nivel de satisfacción de los alumnos, la encuesta también pretende analizar la distribución del tiempo entre la parte presencial y online de las asignaturas híbridas analizadas. En general, casi el 80\% de los estudiantes considera que estas asignaturas deberían contar con una mayor cantidad de horas de enseñanza presencial. La Tabla 3 muestra los principales motivos que estarían vinculados con este mayor peso de la parte presencial de la asignatura híbrida. Como se desprende de los datos recogidos en esta tabla, las principales causas que explicarían esta preferencia se relacionan con la posibilidad de contar con mayor tiempo para explicar conceptos prácticos de la asignatura y para aprender a gestionar más eficientemente el tiempo del alumno. Las 
opiniones vertidas en el focus group estuvieron todas orientadas en el mismo sentido.

Tabla 3

\begin{tabular}{l|c|c}
\hline \hline Mayor número de horas & $\begin{array}{c}\text { Acuerdo } \\
\text { presenciales para: }\end{array}$ & $\begin{array}{c}\text { Desacuerdo } \\
\text { (\%) }\end{array}$ \\
\hline $\begin{array}{l}\text { Más explicaciones prácticas } \\
\text { Aprender a gestionar mis }\end{array}$ & 94,7 & 5,3 \\
recursos más eficientemente & 90,1 & 9,9 \\
$\begin{array}{l}\text { Más práctica de la asignatura } \\
\text { Más trabajo individual con el }\end{array}$ & 88,5 & 11,5 \\
profesor & 85,5 & 14,5 \\
$\begin{array}{l}\text { Adquirir experiencia de trabajo } \\
\text { en equipo }\end{array}$ & 81,7 & 18,3 \\
$\begin{array}{l}\text { Tener más relación con mis } \\
\text { compañeros }\end{array}$ & 79,4 & 20,6 \\
Más explicaciones teóricas & 75,6 & 24,4 \\
\hline \hline
\end{tabular}

\section{CONCLUSIONES}

Entendemos que en toda innovación educativa, tanto metodológica como de entorno docente, la sostenibilidad debe de ser un objetivo esencial, de forma que, como indica Arquero (2012), con el paso del tiempo "deje de ser una innovación y se convierta en la forma normal de hacer las cosas". En ese sentido, en este trabajo no solo se aportan evidencias de las preferencias de los alumnos por determinados ambientes de aprendizaje (el entorno híbrido frente al exclusivamente online), sino que además se investiga sobre las causas de ello, lo que contribuye a dar luz sobre cuáles son las condiciones y características más apreciadas por los estudiantes. Adicionalmente se busca también conocer la preferencia sobre la metodología utilizada en el ambiente híbrido de enseñanza (FC frente a metodología tradicional) y se investiga sus causas y las condiciones que se consideran más adecuadas. Por tanto, las conclusiones definitivas de este trabajo se orientan a conseguir resultados de forma duradera y que además se puedan poner en práctica en otras universidades, es decir, que sean transferibles. Este aspecto ha sido especialmente considerado al haber sido realizada la investigación en dos universidades diferentes en distintos países.

Los estudiantes nos han transmitido que todas las materias no son igualmente adecuadas para la utilización de una única metodología o un determinado entorno de aprendizaje, sino que su preferencia dependerá también del carácter más o menos cuantitativo de las mismas. Es decir, que el "qué enseñar” y el “cómo enseñar” está íntimamente ligados. Por tanto, una primera recomendación de aplicación se refiere a la contextualización de la utilización del entorno y la metodología a utilizar.

En cuanto a la preferencia del entorno híbrido frente al exclusivamente online se ve mayoritariamente avalado por la posibilidad de trabajo presencial con el docente, para aclarar dudas o contextualizar conceptos más teóricos. Es más, los resultados sugieren que los alumnos echan en falta mayoritariamente más horas de presencialidad y lo expresan tanto en las encuestas como en las reuniones grupales mantenidas. En este sentido, una segunda recomendación se refiere a la necesidad de establecer un porcentaje adecuado de docencia presencial frente a online, así como una estructura en las horas presenciales que tenga en cuenta las preferencias del alumno expresadas en esta investigación.

En relación con estas conclusiones, es importante destacar la relevancia que otorgan los estudiantes a dos cuestiones: la adecuada organización de las sesiones presenciales y la calidad de los materiales que se ofrecen para el estudio online. Por tanto, la siguiente recomendación se refiere a que no solo deben de estar bien proporcionados los dos ambientes que conforman el entorno híbrido sino que éstos deben estar adecuadamente organizados para la correcta implementación de la metodología utilizada: en el tiempo presencial deben llevarse a cabo actividades que cumplan los objetivos marcados para este ámbito (aclarar dudas, profundizar en conceptos complejos, prácticas y casos) y en el estudio online proporcionar materiales adecuados y de gran calidad (videos, lecturas, mapas conceptuales, presentaciones, cuestionarios de autoevalución, etc.).

\section{AgRADECIMIENTOS}

Queremos agradecer a la Universidad Europea de Madrid, al Kendall College y a los profesores que han impartido los cursos de los estudiantes de la muestra, sin cuya colaboración este proyecto no hubiera podido llevarse a cabo. Esta investigación ha sido financiada por el programa "Hybrid Teaching \& Learning Research Grant”, concedido por la Oficina de Investigación de la Unidad de Calidad Académica y Acreditación de la red Laureate Network Office (LNO) que pretende investigar el impacto de los métodos digitales de enseñanza y aprendizaje sobre los resultados educativos.

\section{REFERENCIAS}

Aguilar, S. y Barroso, J. (2015) La triangulación de datos como estrategia en investigación educativa. Píxel-Bit. Revista de Medios y Educación. No 47 Julio 2015. Pag 73-78.

Allen, I. E., Seaman, J., \& Garrett, R. (2007). Blending in: The extent and promise of blended learning in the United States. Needham, MA: Sloan-C.

Arbaugh, J. B. (2010) What Might Online Delivery Teach Us About Blended Management Education? Prior Perspectives and Future Directions. Journal of Management Education 2014, Vol. 38(6) 784 -817.

Arquero, J.L. (2012) Investigación en la docencia de la contabilidad. $X V$ Encuentro AECA. Ofir-Esposende (Portugal). Septiembre 2012.

Aste, M. (2012). La clase al revés (o flipped classroom). Retrieved from:http:/quipus.biz/Clase-alRev\%C3\%A9s--2012-.php_[Acceso 09/02/2017].

Bishop, J. L., \& Verleger, M. A. (2013, June). The flipped classroom: a survey of the research. In ASEE National Conference Proceedings, Atlanta, GA (Vol. 30, No. 9).

Bisquerra, R. et al. (2012) Metodología de la investigación educativa. 3rd ed. Madrid: La Muralla.

Blair, E., Maharaj, Ch., Primus, S. (2016). Performance and perception in the flipped classroom. Education and 
Information Technologies, vol. 21, pp. 1465-1482. doi: 10.1007/s10639-015-9393-5.

Butt, A. (2014). Student views on the use of a flipped classroom approach: evidence from Australia. Business Education \& Accreditation, vol. 6, n. 1, pp. 32-43.

Çakiroglu, Ü. \& Öztürk, M. (2017) Flipped Classroom with Problem Based Activities: Exploring Self-regulated Learning in a Programming Language Course. Journal of Educational Technology \& Society, vol. 20, no. 1, pp. 337-349.

Chen, Yuh-Tyng and Chen, Lin-Fan (2016, 22-24 sept). Effects of the Flipped Classroom Model on Student Performance for Vocational College Students. International Conference on Educational Innovation through Technology (EITT). New York: IEEE. doi: 10.1109/EITT.2016.30.

Chuang, H.-H., Weng, C.-Y. and Chen, C.-H. (2016), Which students benefit most from a flipped classroom approach to language learning? British Journal of Education Technology. doi:10.1111/bjet.12530.

Duart, J.M., Gil, M, Pujol, M., Castaño, J. (2008). La universidad en la sociedad red. Barcelona. UOC / Ariel.

Dziuban, Ch., Hartman, J.; Moskal, P. (2004) Blended Learning. EDUCASE Centre for applied research. Research Bulletin. Vol 2004, issue 7.

Garrison, D. R., \& Kanuka, H. (2004). Blended learning: Uncovering its transformative potential in higher education. The Internet and Higher Education, 7, 95105.

Graham, C. R. (2006). Blended learning systems: Definition, current trends, and future directions. In C. J. Bonk \& C. R. Graham (Eds.), The handbook of blended learning: Global perspectives, local designs (pp. 3-21). San Francisco, CA: Pfeiffer.

Hernández Naclares, N. and Pérez Rodríguez, M. (2016).Students' Satisfaction with a Blended
Instructional Design: The Potential of Flipped Classroom. Higher Education. Journal of Interactive Media in Education, 2016(1): 4, pp. 1-12, doi: 10.5334/jime.397.

Llorente, M.C. y Cabero-Almenara, J. (2008) Del eLearning al Blended Learning: nuevas acciones educativas. Quaderns digitals: Revista de Nuevas Tecnologías y Sociedad. IssN-e 1575-9393, n 51.2008.

Mazur, E. (1997). Peer Instruction: A User's Manual. New Jersey: Pearson. ISBN 9780135654415.

Osorio, L. (2010). Características de los ambientes híbridos de aprendizaje: estudio de caso de un programa de posgrado de la Universidad de los Andes. RUSC. Universities and Knowledge Society Journal, ISSNe 1698-580X, Vol. 7, No. 1, 2010

Rotellar, C., Pharm D. \& Cain, J. (2016), Research, Perspectives, and Recommendations on Implementing the Flipped Classroom, American Journal of Pharmaceutical Education, vol. 80, no. 2, pp. 1-9.

Rutherfoord, R. H. and Rutherfoord, J. K. (2013). Flipping the classroom - Is for you? Proceedings of the 14th annual ACM SIGITE conference on Information technology education. Orlando, Florida, USA - October 10 - 12. pp. 19-22 ISBN: 978-1-4503-2239-3; doi:10.1145/2512276.2512299.

Wolff, L-C. y Chan, J. (2016). Flipped Classroom for Legal Education. Singapore: Springer. ISBN 978-981-100478-0 ISBN 978-981-10-0479-7. ISSN 2192-855X ISSN 2192-8568 (electronic) (eBook). doi : 10.1007/978-981-10-0479-7.

Zuber, W. J. (2016). The flipped classroom, a review of the literature. Industrial and Commercial Training, Vol.48 Iss 2 pp. 97-103. ISSN 0019-7858 Doi: 10.1108/ICT-052015-0039. 